\title{
El uso de la Realidad Aumentada en ANL (asignaturas no lingǘsicicas): cicómo didactizar el Sistema Solar en alemán sin conocimientos previos?
}

\author{
Jesuís Martín Cardoso \\ I.E.S. El Argar (Almería)
}

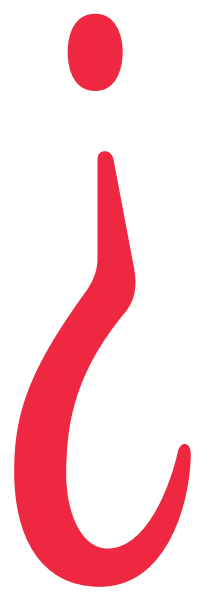

Qué es la Realidad Aumentada?

Frente a la realidad virtual, que es una tecnología que replica un conjunto de escenas u objetos de apariencia o comportamiento real, pero que sólo existe en el código binario de un servidor, la Realidad Aumentada es un sistema que combina elementos del mundo real con información disponible en el mundo digital.

¿Te acuerdas de aquellos adolescentes y no tan adolescentes - que se daban de morros buscando una saga de bichitos inexistentes? PoKemon Go. Pues eso es la Realidad Aumentada.

Probablemente te estés preguntando qué tiene que ver Pokemon Go con la educación. Con razón. No busques en esta aplicación material didáctico porque no lo vas a encontrar. Pero imagina, por un instante, que esos gusarapos disonantes se pudiesen cambiar por personajes históricos, fórmulas matemáticas o locuciones idiomáticas; entonces entenderías el binomio Realidad Aumentada y didactización que quiero presentarte en este artículo.

La Realidad Aumentada logra enriquecer un objeto real a través de la implementación de contenido extraído del mundo digital. Esta técnica abre, pues, un acceso a la incorporación de archivos multimedia que permite un uso inmediato en el aula.

\section{¿Qué es un Código QR?}

La Realidad Aumentada comprende un sinfín de aplicaciones y herramientas. En este caso trabajaré con una técnica sencilla, flexible y resultona: los Códigos QR. En primer lugar porque estamos rodeados de ellos. Un código QR es un código de barras bidimensional cuadrado que puede almacenar datos codificados.

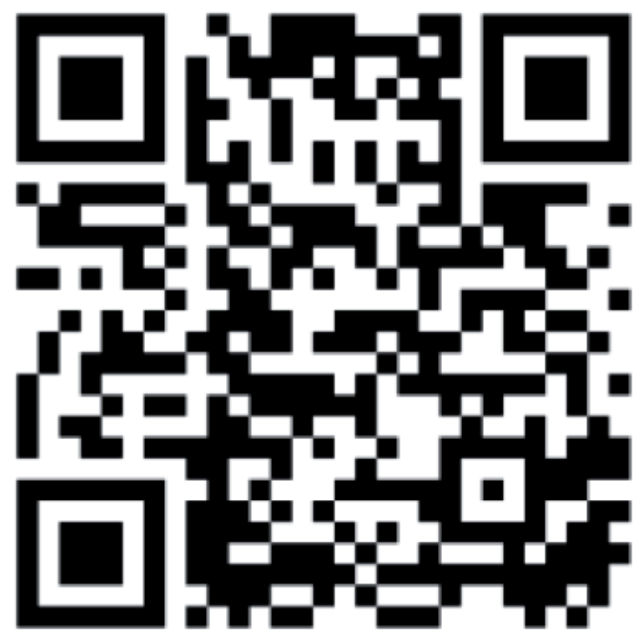

Actualmente los código QR sustituyen a los códigos de barras, almacenando información sobre productos, inventarios, actividades culturales, eventos deportivos, etc. Por regla general los códigos QR contienen un enlace web que permiten obtener más información sobre el soporte físico sobre el que se encuentra, aunque en ocasiones el 
código redirige hacia un archivo listo para ser descargado (pdf, vídeo, etc.)

En segundo lugar porque las herramientas necesarias son muy intuitivas y de código abierto:

- Un lector de códigos QR

- Un generador de códigos QR

\section{Busco proyecto educativo}

¿Tienes ya estas aplicaciones instaladas en tu móvil, tablet $\mathrm{u}$ ordenador? Perfecto. Mi propuesta didáctica es realizar una maqueta del Sistema Solar implementada con Códigos QR. Este proyecto lo llevo a cabo para la sección bilingüe alemán del I.E.S. El Argar con alumnos de $1^{\circ}$ de ESO. Su finalidad es que el alumnado trabaje las destrezas lingüísticas a través del proyecto. Como hándicap cuento con que mis alumnos se enfrentan por primera vez al alemán como primer idioma y el escaso conocimiento de herramientas digitales.

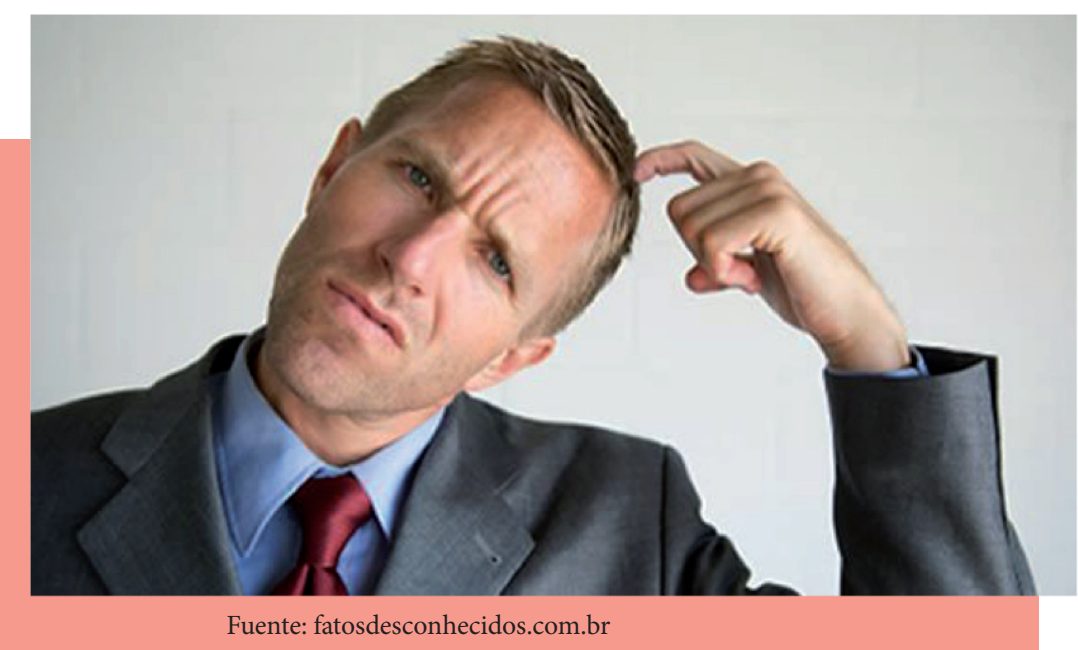

\section{Selección del tema y planteamiento de la pregunta guía}

Cuando abro un proyecto nuevo selecciono un tema ligado a la realidad de los alumnos. En este caso he utilizado el Sistema Solar; se trata de un contenido que ya conocen y me permite ofrecerles una base sólida desde la que trabajar nuevos elementos. Acto seguido planteo una batería de preguntas abiertas que sirven de gancho y me permiten detectar los conocimientos previos sobre el tema.

1. ¿Por qué son redondos todos los planetas?

2. ¿Cuántos planetas en el Sistema Solar tienen anillos?

3. ¿Cómo obtuvieron los planetas sus nombres?

4. ¿Cómo permanecen los planetas en órbita alrededor del Sol?
5. Dónde está la montaña más alta en nuestro sistema solar?

6. ¿Qué planeta es el más rápido en girar sobre sí mismo?

7. ¿Qué planeta tiene los vientos más fuertes?

8. ¿Por qué los planetas tienen diferentes colores?

No te aventures a un proyecto nuevo sin preparación previa. Plantea desde el principio un reto real a tu alumnado. Una buena batería de preguntas le sirve como ejercicio de calentamiento y es un modo ideal para introducirlos en el tema.

\section{Formación de equipos}

En este punto las instrucciones son básicas: grupos de cuatro alumnos, diversidad de perfiles y desempeño de diferentes roles. No suelo participar de manera explícita en la selección de grupos, prefiero arriesgarme y cedo el control en este punto a los alumnos, aunque siempre conservo mi derecho a veto para equilibrar los grupos descompensados. Con respecto a la división de roles tienen que repartirse las siguientes áreas:

1. Dirección: responsable de informar al profesor de los avances del proyecto y gestiona la entrega del mismo en la fecha convenida.

2. Secretaría: responsable de anotar los acuerdos del grupo y las tareas asignadas a cada uno de los miembros.

3. Investigación: responsable de recoger la información, entregarla e informar al resto del grupo de las propuestas de mejoras esbozadas por el profesor.

4. Factoría: responsable de las herramientas, utensilios y material necesario para llevar a cabo el trabajo.

La introducción de diversos roles en un trabajo por equipo concede protagonismo a tu alumnado. Los roles no están previamente marcados $y$, a veces, funciona la psicología inversa. Deja la dirección en manos de alumnos disruptivos. Te sorprenderás, y gratamente.

\section{Definición del producto o reto final}

Este proceso se estructura en torno a la ejecución y presentación de un producto final. Suelo trabajar con productos finales porque es enriquecedor y aumenta la estima de los alumnos. En mi caso, valoro más el recorrido y la adquisición del conocimiento, desde el punto de vista del alumnado es más interesante acabar el proyecto con un elemento tangible. 
El reto final de este proyecto es la creación de una maqueta del Sistema Solar. Esta maqueta incluirá códigos QR en el que los alumnos deberán explicar las características principales de cada uno de los planetas del Sistema Solar en alemán.

No desesperes. En tu caso inténtalo con una infografía, un mural, un rincón interactivo, etc... . Busca un producto que sea vistoso y sencillo, si es la primera vez que pruebas con la Realidad Aumentada.

Llegados a este punto les presento, en la pizarra digital si es posible, la rúbrica del proyecto. La ventaja de trabajar con rúbricas es que el alumnado sabe de antemano cuáles son los criterios con los que su proyecto será evaluado. se solapan y acabo prescindiendo de aquellos que, por cuestiones de tiempo, colindan con las evaluaciones, etc.

:) Mal, muy mal, por mi parte. Necesito incluir un calendario que vertebre la ejecución de mis proyectos. Te prometo que he intentado de todo. Resultado: un desastre. Si algún día encuentro la solución, escribiré un nuevo artículo.

\section{Elaboración del producto}

La idea fundamental de este procedimiento es que el alumno sea responsable de su aprendizaje, mientras que el docente se convierta en un mero guía. Para conseguir esta condición se debe allanar lo máximo posible el camino que les lleve a la consecución del objetivo.

\begin{tabular}{|c|c|c|c|c|}
\hline Categorias & Experto $(10-9)$ & Avanzado (8 - 7) & Aprendiz (6 - 5) & Novel $(4-1)$ \\
\hline $\begin{array}{l}\text { Calidad de la } \\
\text { maqueta }\end{array}$ & $\begin{array}{l}\text { La maqueta muestra una } \\
\text { considerable atención en su } \\
\text { construcción. Sus componentes } \\
\text { están bien presentados y con } \\
\text { detalles originales. No hay marcas, } \\
\text { rayones o manchas de pegamento y } \\
\text { no cuelga nada de los bordes }\end{array}$ & $\begin{array}{l}\text { La maqueta muestra una } \\
\text { considerable atención en su } \\
\text { construcción. Todos los planetas están } \\
\text { cuidadosamente fijados. Sus } \\
\text { componentes están bien presentados } \\
\text { y con muchos detalles. Tiene marcas, } \\
\text { rayones o manchas de pegamento y } \\
\text { no cuelga nada de los bordes }\end{array}$ & $\begin{array}{l}\text { La maqueta muestra poca } \\
\text { atención en su construcción. } \\
\text { Algunos planetas están fijados } \\
\text { al expositor. Sus componentes } \\
\text { están bien presentados y con } \\
\text { muchos detalles. Hay marcas, } \\
\text { rayones o manchas de } \\
\text { pegamento y no cuelga nada } \\
\text { de los bordes }\end{array}$ & $\begin{array}{l}\text { La maqueta muestra falta } \\
\text { atención en su construcción. } \\
\text { Los elementos no están fijados } \\
\text { al expositor Sus componentes } \\
\text { no están bien presentados y } \\
\text { faltan detalles. Hay marcas, } \\
\text { rayones o manchas de } \\
\text { pegamento y cuelgan } \\
\text { elementos de los bordes }\end{array}$ \\
\hline Creatividad & $\begin{array}{l}\text { Varios de los objetos usados en la } \\
\text { maqueta reflejan un grado de } \\
\text { creatividad excepcional }\end{array}$ & $\begin{array}{l}\text { Uno o dos objetos usados en la } \\
\text { maqueta reflejan un grado de } \\
\text { creatividad excepcional }\end{array}$ & $\begin{array}{l}\text { Uno objeto usado en la } \\
\text { maqueta está personalizado }\end{array}$ & $\begin{array}{l}\text { Los objetos no denotan } \\
\text { creatividad ni atractivo }\end{array}$ \\
\hline Código QR & $\begin{array}{l}\text { Todos los códigos QR se } \\
\text { corresponden con los elementos } \\
\text { señalados y recogen una descripción } \\
\text { clara y precisa }\end{array}$ & $\begin{array}{l}\text { La mayoría de los códigos QR } \\
\text { corresponden con los elementos } \\
\text { señalados y recogen una descripción } \\
\text { clara y precisa }\end{array}$ & $\begin{array}{l}\text { Alguno de los códigos QR } \\
\text { corresponden con los } \\
\text { elementos correspondientes y } \\
\text { recogen una descripción clara } \\
\text { y precisa }\end{array}$ & $\begin{array}{l}\text { Muchos de los códigos QR no } \\
\text { corresponden a los elementos } \\
\text { señalados y no recogen una } \\
\text { descripción clara y precisa }\end{array}$ \\
\hline Presentación & $\begin{array}{l}\text { Los autores del trabajo, el título, } \\
\text { la asignatura y el curso aparecen } \\
\text { señalados en la maqueta en un lugar } \\
\text { visible }\end{array}$ & $\begin{array}{l}1 \text { de los elementos señalados } \\
\text { anteriormente no aparecen en la } \\
\text { maqueta en un lugar visible }\end{array}$ & $\begin{array}{l}2 \text { de los elementos } \\
\text { señalados anteriormente no } \\
\text { aparecen en la maqueta en un } \\
\text { lugar visible }\end{array}$ & $\begin{array}{l}30 \text { más elementos } \\
\text { señalados anteriormente no } \\
\text { aparecen en la maqueta en un } \\
\text { lugar visible }\end{array}$ \\
\hline
\end{tabular}

Si aún no has trabajado con rúbricas, te las recomiendo. Son sencillas de producir y, nivelando adecuadamente los criterios, son muy útiles para conducir el trabajo en equipo dentro del aula. Un truco: para crear una rúbrica comienza por el nivel experto.

\section{Planificación}

Tengo que reconocerlo, este es mi talón de Aquiles. Lo ideal sería un plan de trabajo donde se especifican tareas previstas, áreas encargadas y calendario de entregas. Mi respuesta: imposible. En ninguno, repito, en ninguno de los proyectos que gestiono, he sido capaz de atenerme a un plan, calendario o sistema de organización. Los proyectos
Como puedes comprobar, estamos ante el núcleo central del proyecto. Este punto requiere precisión milimétrica y pulso de cirujano. Te recomiendo que repitas hasta la extenuación los pasos que el alumnado debe realizar para completar el reto.

Los grupos deben tener muy claro cuál es el producto final que tienen que presentar: una maqueta del Sistema Solar implementada con códigos QR.

El modelo en cascada de Royce (1970) nos aconseja que para desarrollar un software hay que ordenar rigurosamente las etapas del proceso de desarrollo, de tal forma que el inicio de cada etapa debe esperar a la finalización de la etapa anterior. Este modelo permite una implementación en el aula muy intuitiva. 


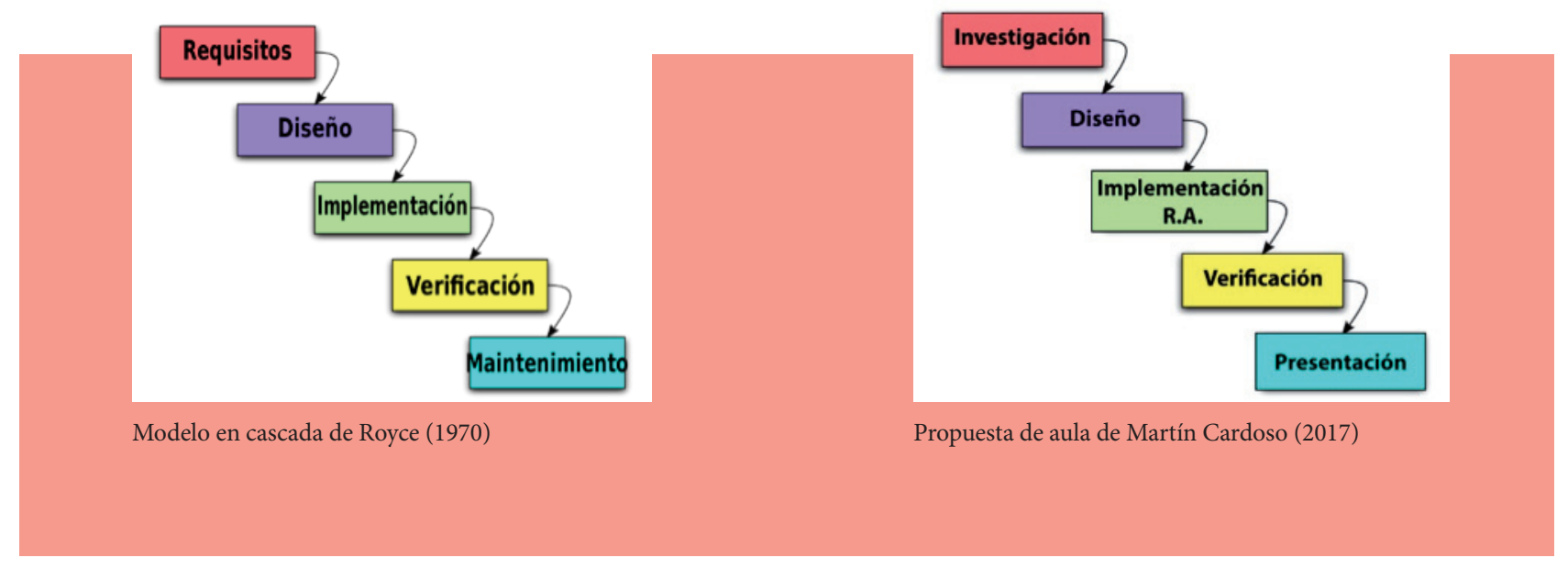

1. Investigación: en esta fase se ofrecen al alumnado una serie de páginas web desde las cuales podrán obtener la información necesaria para recopilar las características principales de nuestro Sistema Solar.

2. Diseño: el alumnado debe presentar un diseño de la maqueta del Sistema Solar. Una vez aprobado podrán comenzar desarrollarla.

3. Implementación R.A.: se incrustan los códigos QR con la información en alemán. Como este es el paso más complicado para el alumnado, le dedicaré un apartado especial para explicar cómo se lleva a cabo en mis proyectos.

4. Verificación: una vez implementados los códigos QR debe verificarse que todos ellos funcionen y se encuentren en la posición correspondiente.

5. Presentación: los alumnos deben presentar su proyecto describiendo su fase de construcción, problemas que se han encontrado y posibles soluciones.

\section{Implementación de los códigos QR}

Los códigos QR son la médula de este proyecto. Un código QR no es más que una imagen, que, utilizando el programa adecuado, te redirecciona a un enlace. Estamos acostumbrados a que el enlace final de los códigos QR sea un producto comercial; pero en este caso se trata de un producto educativo. Este producto educativo trabaja tres de las cuatro habilidades lingüísticas: comprensión lectora, comprensión auditiva y expresión oral. Recuerda que mis alumnos de $1^{\circ}$ ESO no han tenido previo contacto con el idioma alemán. Y yo pretendo que introduzca información en alemán sobre cada planeta del Sistema Solar en la susodicha maqueta. O empiezo a sacar ases de la manga o pierdo la partida:
- As de corazones: es imprescindible que los alumnos se apoyen en un texto previo donde se haya trabajado con la información. El ejercicio que les propongo es un texto donde tienen que rellenar los huecos que aparecen en él. También cuento con el apoyo del departamento de alemán quien repasa y asume los contenidos gramaticales.

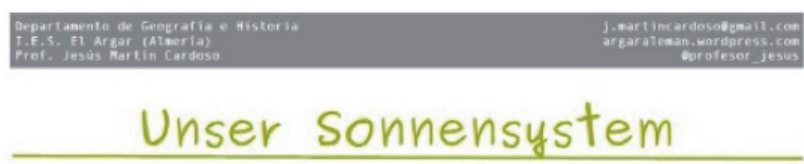

Füll die Lücke aus und findet die richtige Lösung

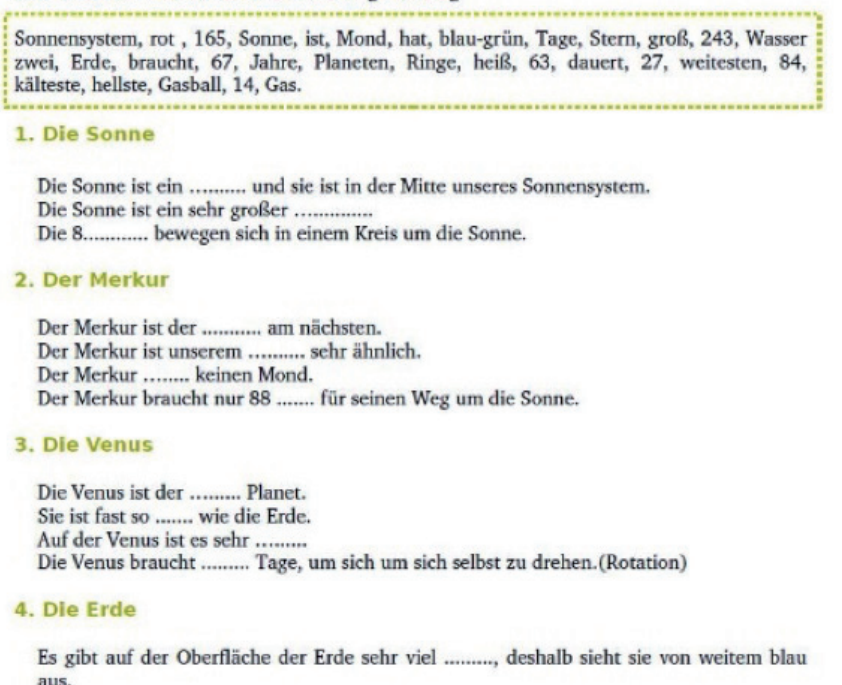
aus.

Fragmento del texto con hueco que se le ofrece al alumnado para completar el ejercicio. Gracias a este ejercicio mi alumnado trabaja la comprensión lectora. Para acceder al texto completo solamente tienes que clickear sobre la siguiente dirección: http://bit.ly/textoSistemaSolar

- As de picas: en este caso, la lectora se ofreció amablemente a grabar el texto completo en alemán. Mi consejo es subir la grabación a una plataforma 
online, de esta forma el alumnado puede acceder en todo momento a los datos y corregir tanto el texto como mejorar su pronunciación.

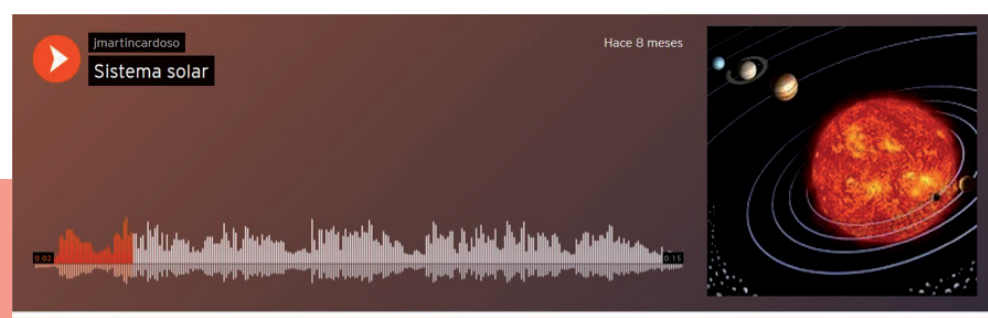

Canal de Soundcloud donde se almacena la grabación con la información sobre los planetas del Sistema Solar. http://bit.ly/sonidoSistSolar

Utilicé la aplicación Soundcloud como base para almacenar la grabación online. De esta forma me aseguro de que mi alumnado está trabajando las competencias auditiva.

- As de tréboles: para reproducir el texto los alumnos deben crear una cuenta en Soundcloud y subir su grabación a esta plataforma digital. Menos mal que en mi centro me dejan trabajar con móviles, sin la ayuda de estos dispositivos este paso sería aún más complejo.

- As de diamantes: para generar un código QR necesitas un enlace al que este redirigirá y una aplicación online para crearlo. Los alumnos obtienen el enlace de la plataforma en la que han subido sus grabaciones. La aplicación online para generar el código se llama Unitag. Te nombro esta porque la más versatil y flexible que he encontrado.

Ahora es cuando los alumnos tienen que colocar la url de la grabación en el correspondiente cajetín, validar e imprimir.

En la mayoría de los casos aparecen con un pendrive y soy yo quien se encarga de imprimir los códigos $Q R$. Es en ese momento donde me pregunto por qué me meto yo en este berenjenal; aunque luego se me pasa.

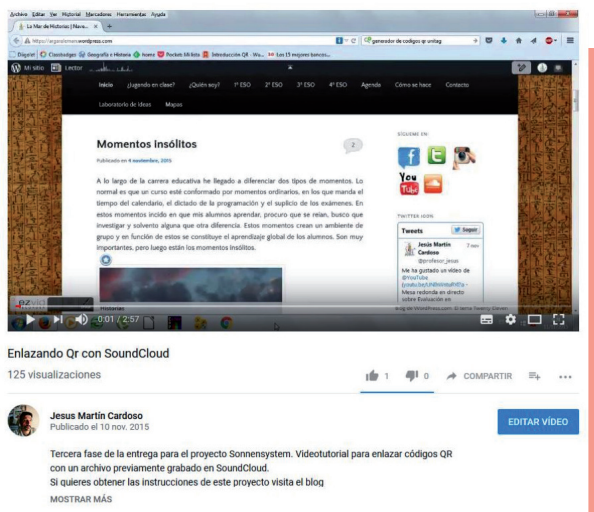

Videotutorial: enlazando QR con SoundCloud. Tras años de observando que este es precisamente el paso que más complicaciones genera a los alumnos, creé un videotutorial para evitar explicar uno a uno, año tras año cómo se crea un código QR. Échale un vistazo y verás qué sencillo. Clickea sobre la siguiente dirección y accederás al vídeo: http://bit.ly/generarQR

\section{La guía}

Por mucha ayuda que sea el modelo en cascada te aseguro que más de un alumno se perderá o se saltará los pasos. Tampoco hay que tenérselo en cuenta, son alumnos de $1^{\circ}$ de ESO aprendiendo a crear códigos $Q R$.

La solución es crear un guía que les permita reiniciar el proceso en caso de problemas. Además de servir de apoyo para el propio docente.

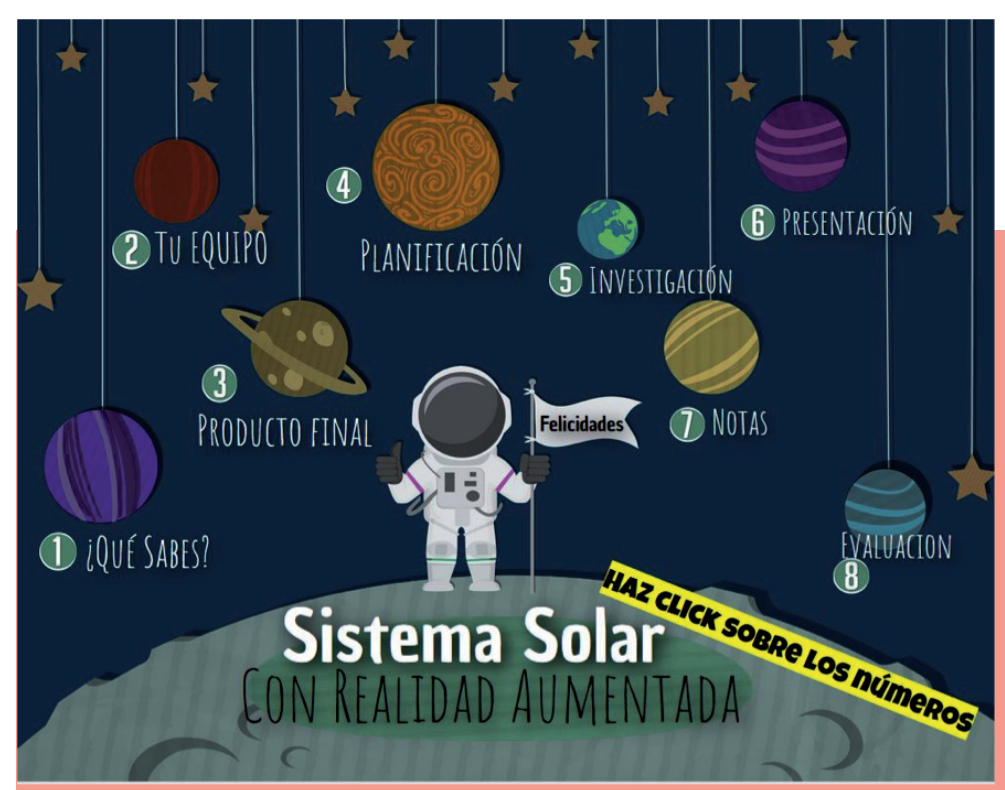

Presentación de la guía para el alumnado. Clickea aquí para verla: http://bit.ly/abpSisSolar

\section{A modo de conclusión}

El objetivo principal de esta propuesta era crear una guía para la utilización de códigos QR en las ANL (en este caso alemán). Para mostrarte un ejemplo he utilizado como soporte físico una maqueta, aunque podría haber sido otro cualquiera. La dificultad de la utilización de códigos QR tiene más que ver con su concepción que con su manejo técnico. Es una técnica asequible que consiste en introducir una URL en un generador de códigos. Cualquier contenido multimedia de la red de redes o creado ex professo para este proyecto puede ser incluido. Ahora es cuestión de que busques un soporte adecuado y diseñes el modelo de información. Lo demás es copiar $(\mathrm{Ctrl}+\mathrm{C}$ ) y pegar (Ctrl V). 
Cózar Gutiérrez, R. / De Moya Martínez, M. V. / Hernández Bravo, J. A. / Hernández Bravo, J. R (2015): “Tecnologías emergentes para la enseñanza de las Ciencias Sociales. Una experiencia con el uso de Realidad Aumentada en formación inicial de maestros", Digital Education Review 27, pp. 138-153. http://greav.ub.edu/der [último acceso: 4 de junio de 2018].
Cózar, R. / Guerrero, O. (2013): 'La Realidad Aumentada en la enseñanza/aprendizaje de las Ciencias Sociales". En Maquilón, J.J. / Orcajada, N. (eds.): Investigación e innovación en formación del profesorado. Murcia. Editum.

Fundación Telefónica (2011): "Realidad aumentada: una nueva lente para ver el mundo", https://www. fundaciontelefonica. com/arte_cultura/publicaciones-listado/ pagina-item-publicaciones/itempubli/80/ [último acceso: 4 de junio de 2018].
Moreno, J.R. (2016): “Geovicente: la enseñanza de Geografía con códigos QR'. En Tortosa, M.T./ Álvarez, J.D./ Pellín, N. (Coords): XIV Jornadas de investigación en docencia universitaria, Alicante: Ediciones ICE, pp. 193-209.

Royce, Winston (1970): "Managing the Development of Large Software Systems", Proceedings IEEE. WESCON, Los Angeles, 25-28/8/1970, pp. 328-338.
Publireportaje de los autores Brigitte Braucek y Andreu Castell

\section{Gramática básica del alemán}

Dieciséis años después de la publicación de la Gramática de la lengua alemana (1997), complementada en el año 2000 con un volumen dedicado a los ejercicios correspondientes, apareció en 2013 la Gramática básica del alemán. Se trata de un manual pensado para los niveles A1, A2 y B1 fijados por el Marco Común Europeo de Referencia para las Lenguas. Concebida expresamente para hablantes del español, ofrece compara- ciones explícitas entre el alemán y el español siempre que se considera conveniente para facilitar la comprensión.

\section{Contiene numerosos} $y$ variados ejercicios integrados en las explicaciones, cuya solución se ofrece al final del libro, todos los ejemplos están traducidos y el vocabulario de los mismos y de los ejercicios pertenece exclusivamente a los niveles mencionados. El usuario dispone de varias formas de acceder a la información deseada: puede hacerlo consultando el índice general situado al inicio del libro, pero también vía el índice alfabético de materias y palabras que se ofrece al final. La terminología gra- matical utilizada se corresponde con la terminología tradicional que conocen los usuarios españoles de sus estudios en la enseñanza primaria y secundaria.

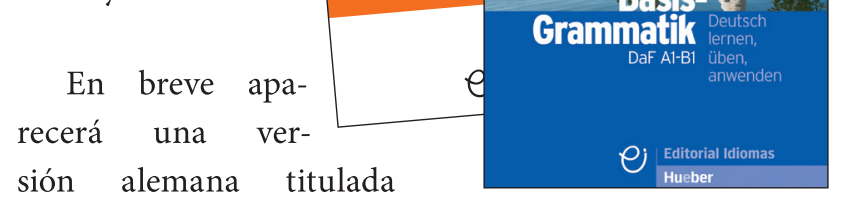

\section{Basisgrammatik DaF A1-B1,}

Deutsch lernen, üben, anwenden. Está redactada en alemán fácil, es decir que se caracteriza por frases cortas, vocabulario básico y estructuras lo más sencillas posibles y se dirige a un usuario universal, por lo que también puede ser usado por españoles que quieran lanzarse a la aventura de consultar una gramática básica redactada en alemán.

Y, tras la tan favorable acogida que ha tenido la versión en español, se están preparando adaptaciones al portugués y al italiano, entre otros idiomas...

Editorial Idiomas

Hueber Verlag 